\title{
Inhibition of miR-96 expression reduces cell proliferation and clonogenicity of HepG2 hepatoma cells
}

\author{
DONG XU ${ }^{1,2}$, XINGXING HE $^{1}$, YING CHANG ${ }^{1}$, CHUANRUI XU $^{3}$, \\ XIANG JIANG ${ }^{1}$, SHUZHEN SUN ${ }^{1}$ and JUSHENG LIN ${ }^{1}$ \\ ${ }^{1}$ Institute of Liver Diseases, Tongji Hospital, Tongji Medical College, Huazhong University of Science \\ and Technology, Wuhan, Hubei 430030; ${ }^{2}$ Department of Senile Disease, The 161 th Central \\ Hospital of PLA, Wuhan, Hubei 430010; ${ }^{3}$ School of Pharmacy, Tongji Medical College, \\ Huazhong University of Science and Technology, Wuhan, Hubei 430030, P.R. China
}

Received August 6, 2012; Accepted October 17, 2012

DOI: $10.3892 /$ or.2012.2138

\begin{abstract}
RNAs (miRNAs) are negative regulators of gene expression and can function as tumor suppressors or oncogenes. Several miRNAs are associated with the development of hepatocellular carcinoma (HCC). miR-96 has been closely associated with cell proliferation and clonogenicity. Upregulation of miR-96 has been observed in various types of cancer. However, the biological function of miR-96 in hepatocarcinogenesis remains largely unknown. In this study, we demonstrated that miR-96 was upregulated in HCC and inhibition of miR-96 significantly suppressed HCC cell proliferation and colony formation. The expression levels of forkhead box O1 (FOXO1) and forkhead box O3a (FOXO3a) were upregulated when miR-96 was inhibited in HCC cells and the inhibition of FOXO1 and FOXO3a promoted HCC cell proliferation and colony formation. Collectively, these data reveal an important contribution of miR-96 to hepatocarcinogenesis and suggest a role for FOXO1 and FOXO3a dysregulation in this process. Thus, the use of a synthetic inhibitor of miR-96 may be a promising approach for the treatment of HCC.
\end{abstract}

\section{Introduction}

Hepatocellular carcinoma (HCC), the major type of liver cancer, is the fifth most frequent neoplasm and the third most common cause of cancer-related mortality in the world, particularly in Asia and sub-Saharan Africa (1). Epidemiological studies have demonstrated that hepatitis $B$ virus (HBV) infection is one of

Correspondence to: Dr Xingxing He or Dr Jusheng Lin, Institute of Liver Diseases, Tongji Hospital, Tongji Medical College, Huazhong University of Science and Technology, Wuhan, Hubei 430030, P.R. China E-mail: xxhe2012@126.com

E-mail: jslin@tjh.tjmu.edu.cn

Key words: miR-96, microRNA, hepatocellular carcinoma, forkhead box $\mathrm{O}$ the key risk factors for HCC (2), especially in eastern Asia. In western countries where hepatitis B infections are rare, however, risk factors for $\mathrm{HCC}$ include cirrhosis due to alcohol consumption or hepatitis $\mathrm{C}$ virus (HCV) infection $(3,4)$.

microRNAs (miRNAs) are evolutionarily-conserved, small, non-coding RNA molecules, approximately 22 nucleotides in length, which are cleaved from a 70- to 80-nt partially duplexed precursor (pre-miRNA). miRNAs typically regulate post-transcriptional gene expression by interacting with sequences within the 3'-untranslational region (3'-UTR) of the target mRNA and play important roles in a variety of biological processes, including cell cycle regulation, cell differentiation, proliferation and apoptosis $(5,6)$. Accumulating evidence indicates that miRNAs are extensively involved in the pathogenesis of several types of human cancer (7). Approximately $50 \%$ of the genes that encode miRNAs reside in cancer-associated genomic regions or fragile sites and this fact highlights the importance of miRNAs in tumorigenesis (8).

miR-96 has been recognized as an oncogenic miRNA that is upregulated in various types of cancer (9-11). In HCC, the role of miR-96 remains largely unknown. Recent studies identified the deregulation of miR-96 as being significantly associated with different risk factors for HCC, including HBV infection and alcohol consumption. Moreover, miR-96 was upregulated in several HCC cell lines (12).

The FOXO subfamily of Forkhead transcription factors are key tumor suppressors in mammalian cells (13). To date, suppression of FOXOs in cancer cells was thought to be mainly due to activation of multiple onco-kinases by a phosphorylation-ubiquitylation-mediated cascade. Evidence suggests that members of the FOXO family are regulated by miRNAs. The level of miR-96 was significantly upregulated in endometrial cancer compared with normal endometrium and overexpression of miR-96 effectively downregulated forkhead box O1 (FOXO1) expression in endometrial cancer cells (14). The expression of miR-96 was also markedly upregulated in breast cancer cells and tissues, compared with normal breast epithelial cells and normal breast tissues (11). Further study demonstrated that miR-96 downregulated forkhead box O3a (FOXO3a) expression in breast cancer cells by directly targeting the FOXO3a 3'-UTR (9). 
Based on these findings, we selected miR-96 for further investigation and hypothesized that miR-96 could influence the biological behaviors of HCC cells and affect the expression of FOXO1 and FOXO3a in HCC cells. Our results indicated that downregulation of miR-96 effectively upregulated FOXO1 and FOXO3a expression, and also inhibited cell proliferation and clonogenicity. We concluded that miR-96 may play an important role as an oncogenic miRNA in the development and progression of HCC by regulating the expression of FOXO1 and FOXO3a.

\section{Materials and methods}

Cell culture and transfection. HepG2 cells (ATCC, Manassas, VA, USA) were maintained in Dulbecco's minimal essential medium (DMEM) (Invitrogen Inc., USA), supplemented with $10 \%$ fetal bovine serum (FBS) (Gibco, Grand Island, NY, USA) and $1 \%$ penicillin/streptomycin at $37^{\circ} \mathrm{C}$ under an atmosphere of 5\% carbon dioxide. Normal primary human hepatocytes (PHHCs) were isolated using a modified fourstep retrograde perfusion technique according to the ethical and institutional guidelines and following the informed consent of the tissue donors as previously described (15). The study was approved by the Research Ethics Committee at the Tongji Hospital of Huazhong University of Science and Technology.

The miR-96 inhibitor, anti-miR-96 (a chemically-modified single-stranded nucleic acid targeting miR-96) and the corresponding negative control, anti-miR-NC (a chemicallymodified single-stranded nucleic acid of random sequence) were obtained from Ambion, Inc. (Austin, TX, USA). Small interfering RNAs (siRNAs) targeting FOXO1 and FOXO3a and their negative controls (siR-NC) were purchased from RiboBio Co., Ltd. (Guangzhou, China). The sequences of the FOXO1-specific siRNA (siR-FOXO1) (16) and FOXO3aspecific siRNA (siR-FOXO3a) (17) are listed in Table I. Cells were transfected with siRNA or miRNA inhibitor using Lipofectamine 2000 (Invitrogen, Carlsbad, CA, USA) according to the manufacturer's instruction.

Cell proliferation assay. Cell proliferation was assessed at 24, 48 and $72 \mathrm{~h}$ after transfection using the CellTiter $96^{\circledR}$ AQueous One Solution Cell Proliferation Assay kit (Promega, Madison, WI, USA) as previously described (15).

Flow cytometry analysis of cell cycle and apoptosis. To analyze the effects of siRNA or miRNA inhibitors on the cell cycle, cells were seeded in 6-well plates. Seventy-two hours after transfection, cells were collected and fixed in $70 \%$ ethanol at $4^{\circ} \mathrm{C}$ overnight. After washing and resuspending the fixed cells in $100 \mu \mathrm{l}$ phosphate-buffered saline, they were treated with $50 \mu \mathrm{l} \mathrm{RNase}(50 \mathrm{mg} / \mathrm{ml})$ at $37^{\circ} \mathrm{C}$ for $30 \mathrm{~min}$ and stained with $200 \mu \mathrm{l}$ propidium iodide (KeyGen Biotech. Co., Ltd., Nanjing, China) at $4^{\circ} \mathrm{C}$ for $30 \mathrm{~min}$. Cell cycle analysis was performed using a FACSAria cell sorting system (BD Biosciences, San Jose, CA, USA). For analysis of apoptosis, cells seeded in 24-well plates were harvested $72 \mathrm{~h}$ after transfection and processed with the Annexin V-FITC Apoptosis Detection kit (KeyGen Biotech Co., Ltd.) according to the manufacturer's instructions.
Table I. siRNAs for FOXO1 and FOXO3a.

siRNA name Primer sequence $\left(5^{\prime} \rightarrow 3^{\prime}\right)$

siR-FOXO1

Sense

Antisense

GCGGGCUGGAAGAAUUCAAdTdT

siR-FOXO3a

Sense

Antisense UUGAAUUCUUCCAGCCCGCdTdT

GCACAGAGUUGGAUGACGUdTdT ACGUCAUCCAACU CUGUGCdTdT

Cell migration assay. The migration assay was performed by using Transwell insert chambers $(6.5 \mathrm{~mm}$ in diameter, $8 \mu \mathrm{m}$ pore size; Corning, USA). For the migration assay, $2 \times 10^{5} \mathrm{HepG} 2$ cells, that had been transfected with siRNA or miRNA inhibitor, were placed into the upper chamber in serum-free medium in triplicate $12 \mathrm{~h}$ after transfection. The lower chamber was filled with $600 \mu$ l DMEM with $10 \%$ FBS as the chemoattractant. After incubation for $24 \mathrm{~h}$ at $37^{\circ} \mathrm{C}$ in a humidified incubator with an atmosphere of 5\% carbon dioxide, non-migrating cells in the upper chambers were removed by using a cotton swab, and cells that had migrated to the lower surface of the membrane were fixed with methanol and stained with $0.1 \%$ crystal violet. The cells that had migrated were scored by counting at least six fields per membrane under a light microscope.

Analysis of clonogenicity in vitro. A colony formation assay was used to assess the clonogenicity of HepG2 cells transfected with different miRNAs or siRNAs. To analyze the ability of transfected cells to form colonies, 100 viable HepG2 transfectants were placed in 24-well plates $24 \mathrm{~h}$ after transfection and were maintained in complete medium for 2 weeks. Colonies were fixed with methanol and stained with $0.1 \%$ crystal violet in $20 \%$ methanol. The anchorage-independent growth assay was performed in 24-well plates. Eight hundred cells were trypsinized and suspended in $1 \mathrm{ml}$ complete medium containing $0.3 \%$ low-melting-point agarose (Sigma, St. Louis, MO, USA). The agar-cell mixture was plated on top of a bottom layer with $0.5 \%$ complete medium agar mixture. Two weeks later, colonies were visualized by staining with $0.05 \%$ crystal violet and viable colonies that contained $>50$ cells or were $>0.1 \mathrm{~mm}$ were counted with an ocular micrometer. Each transfectant was seeded into wells in triplicate and the experiment was repeated twice.

\section{Real-time quantitative PCR}

Detection of miR-96 expression. Total RNA was extracted from cells with the mirVana miRNA isolation kit (Ambion). Synthesis of cDNA from $10 \mathrm{ng}$ total RNA was carried out using the TaqMan miRNA Reverse Transcription kit (Ambion) and cDNA was then subjected to TaqMan Quantitative Real-Time PCR using an miR-96-specific TaqMan microRNA assay on an ABI PRISM StepOnePlus Real-Time PCR System (Applied Biosystems, Foster City, CA, USA). Expression levels of the miRNAs were normalized to that of snRNA U6, which served as the internal control.

Detection of mRNA expression. Total RNA was prepared using the mirVana PARIS kit (Ambion) and the reverse tran- 
scription reactions were performed using the First Strand cDNA Synthesis kit (Fermentas, Burlington, ON, Canada). Synthesized cDNA was used as a template for PCR and the quantitative reactions were monitored in real-time using the SYBR Green dye detections with the SYBR Green Premix Reagent (Takara Bio, Inc., Shiga, Japan). The primers used for FOXO1, FOXO3a and $\beta$-actin are listed in Table II and were synthesized by Sangong Biotech Co., Ltd. (Shanghai, China). $\beta$-actin was used as an internal standard for the quantification of the PCR. The relative expression was calculated using the comparative threshold cycle $(\mathrm{Ct})$ method.

Western blotting. Total proteins were harvested with the mirVana PARIS kit (Ambion) and quantified by a BCA protein assay kit (Pierce, Rockford, IL, USA). Cell lysates were separated by electrophoresis, blotted onto membranes $(0.45 \mu \mathrm{m}$; Millipore) and treated with the following primary antibodies: rabbit anti-FOXO1 (1:1,000; Epitomics, Burlingame, CA, USA), rabbit anti-FOXO3a antibody (1:1,000; Epitomics), rabbit anti- $\beta$-actin antibody (1:1,000; Abmart, Shanghai, China). The blots were then incubated with an anti-rabbit secondary antibody conjugated to horseradish peroxidase and visualized by using ECL reagents (Pierce).

Statistical analysis. Data are presented as the means \pm SD. Differences between two groups were evaluated using the Student's t-test. A value of $\mathrm{P}<0.05$ was considered to indicate statistically significant differences.

\section{Results}

miR-96 is upregulated in HepG2 cells and is efficiently inhibited by anti-miR-96. To determine whether miR-96 is upregulated in human HCC, we detected the expression of miR-96 in HepG2 and PHHC cells by real-time PCR analysis. We found that miR-96 was expressed in significantly higher levels in HepG2 cells, compared to the levels in PHHCs (Fig. 1A). This observation is consistent with previous miRNA microarray data that suggested a link between the upregulation of miR-96 and progression to cirrhosis and HCC (12). To investigate the pathophysiological role of miR-96 in $\mathrm{HCC}$, we performed loss-of-function analysis using anti-miR-96 to inhibit endogenous miR-96. miR-96 was inhibited in HepG2 cells by anti-miR-96 (Fig. 1B). These results show that miR-96 expression is indeed increased in HCCs and that anti-miR-96 can effectively block the expression of miR-96.

miR-96 modulates cell proliferation, migration and colony formation of HepG2 cells. Sustained cell growth is a hallmark of cancer. In the HepG2 cell line, transfection with anti-miR-96 markedly decreased cell proliferation (Fig. 1C). This result indicates that the inhibition of miR-96 reduces the growth of HCC cells and suggests that miR-96 is necessary for maintaining the proliferation of HCC cells.

We assessed the role of miR-96 in cell migration, a key determinant of malignant progression and metastasis. Transwell insert chambers were introduced to investigate the impact of miR-96 on cell motility. HepG2 cells were transfected with either the miR-96 inhibitor or a negative control, and vertical migration was assessed. Transfection with
Table II. Primers used for quantitative RT-PCR with SYBR Green dye detection.

Primer name $\quad$ Primer sequence $\left(5^{\prime} \rightarrow 3^{\prime}\right)$

\section{FOXO1}

Sense

Antisense

GACAGCCCTGGATCACAGTTT

FOXO3a

Sense

Antisense

TTCCGTAAGCAAGCCGTGTA

$\beta$-actin

Sense

Antisense

AGAGGGAAATCGTGCGTGAC CAATAGTGATGACCTGGCCGT

anti-miR-96 caused a significant decrease in cell migration, compared with anti-miR-NC transfection (Fig. 1D). This result supports a functional role for miR-96 in mediating cell migration in malignant hepatocytes.

To assess the function of miR-96 in tumor formation, colony formation and anchorage-independent growth were measured in HepG2 cells. Analysis of colony formation showed that cells transfected with anti-miR-96 displayed much fewer and smaller colonies than the control transfectants (Fig. 1E). Inhibition of miR-96 also reduced the anchorage-independent growth of HepG2 cells significantly, as shown by the decrease in the colony number and size (Fig. 1E).

miR-96 affects the cell cycle but does not induce apoptosis of HepG2 cells. To delineate a possible role of miR-96 in cell division, cell cycle regulation and apoptosis FACS analysis, followed by flow cytometry, was carried out. Compared to the negative control, anti-miR-96 transfection caused significant cell cycle arrest (Fig. 2A), but did not induce significant apoptosis (Fig. 2B) in HepG2 cells.

miR-96 inhibits the expression of FOXO1 and FOXO3a. Real-time RT-PCR demonstrated that FOXO1 and FOXO3a mRNA levels were upregulated in anti-miR-96-transfected HepG2 cells compared with cells transfected with the negative control and that the protein levels of FOXO1 and FOXO3a were substantially increased when miR-96 was inhibited (Fig. 2C and D). These results indicate that FOXO1 and FOXO3a might be the targets of miR-96 in HepG2 cells.

FOXO1 and FOXO3a are efficiently inhibited by siRNAs. To assess whether siRNAs could alter the expression of FOXO1 and FOXO3a in HCC cells, we investigated the expression of FOXO1 and FOXO3a in HepG2 cells transfected with FOXO1and FOXO3a-specific siRNAs, or a negative control. Real-time RT-PCR demonstrated that FOXO1 and FOXO3a mRNA levels were downregulated in HepG2 cells transfected with the specific siRNAs, but not with the corresponding negative controls (Fig. 3A) and that the protein level of FOXO1 and FOXO3a were substantially decreased as well (Fig. 3B and C). These results indicate that the expression of FOXO1 and FOXO3a are efficiently decreased by siRNAs in HepG2 cells. 


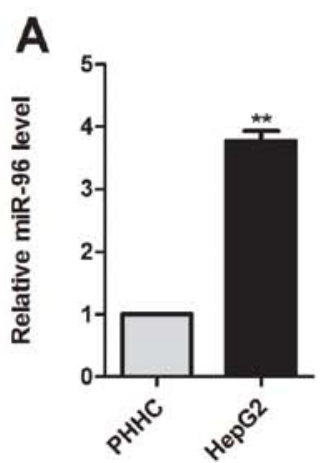

D

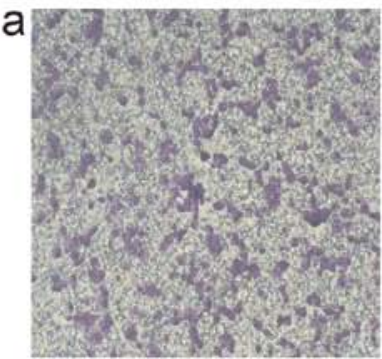

anti-miR-NC

E

a

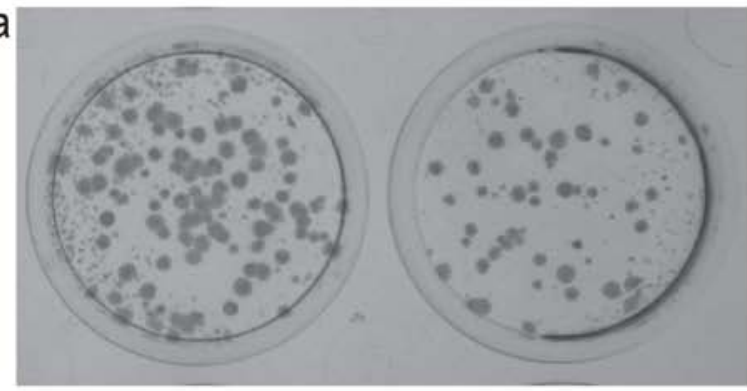

anti-miR-NC

C

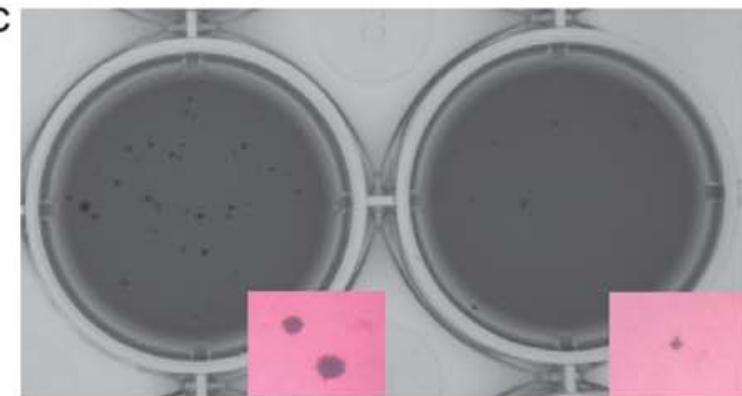

anti-miR-NC
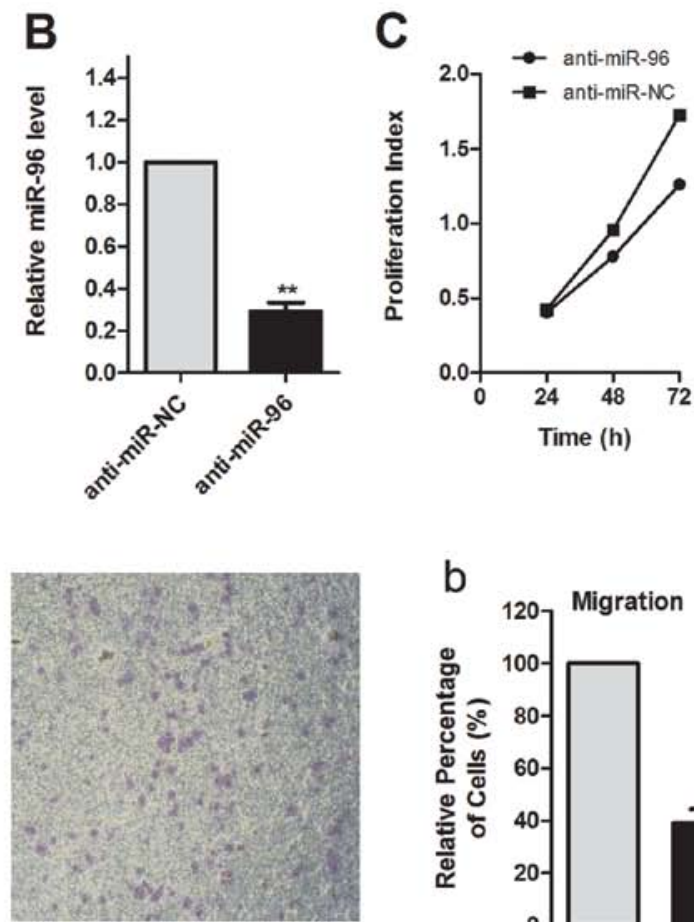

anti-miR-96
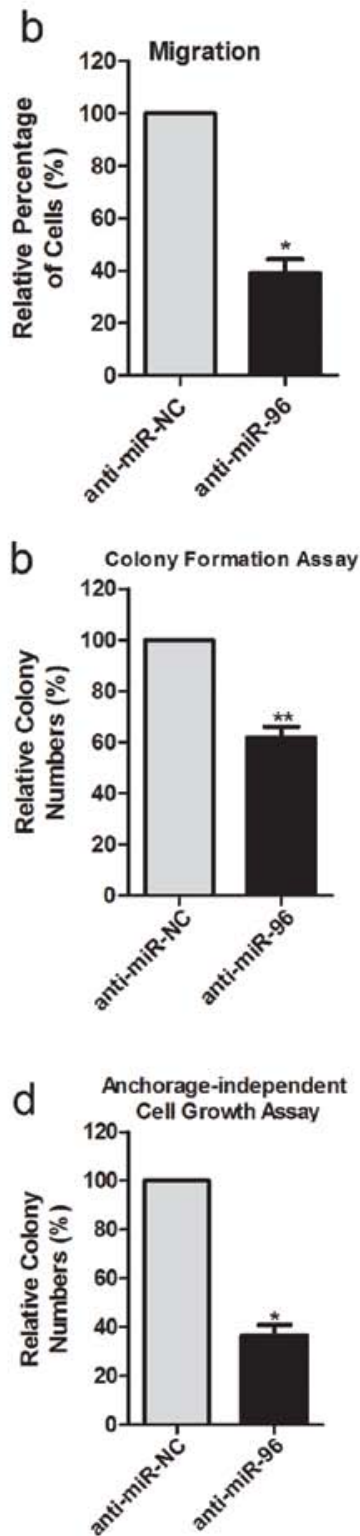

Figure 1. miR-96 is overexpressed in HCC cells and is necessary for their growth, migration and colony formation. (A and B) The level of miR-96 is significantly higher in HepG2 cells compared with PHHC. The expression of miR-96 is effectively inhibited by transfection with anti-miR-96. The expression data were normalized to U6 expression, and the normalized miR-96 level in PHHCs or the anti-miR-NC transfectants was set to 1.0. (C) The cellular proliferation rates were strongly decreased in anti-miR-96 transfected cells compared with the negative control, 24, 48 and $72 \mathrm{~h}$ after transfection. (Da) The results of cell migration across an $8-\mu \mathrm{m}$ pore size membrane without Matrigel. (Db) Histogram of the relative percentage of cells across a membrane with $8-\mu \mathrm{m}$ pores without Matrigel, indicating that the proportion of cells is decreased in the samples transfected with anti-miR-96, compared with those transfected with anti-miR-NC. The relative percentage of migrating cells in the anti-miR-NC-transfected sample was designated as $100 \%$. (Ea and c) Representative results of colony formation and anchorage-independent growth in soft agar. The numbers of colonies and their sizes are strongly decreased in anti-miR-96-transfected samples compared with anti-miR-NC-transfected samples. Each bar corresponds to the means \pm SD of three experiments. (Eb and d) Histograms indicate that anti-miR-96 could markedly inhibit colony formation and anchorage-independent cell growth. The percentage of colony numbers in the negative control was designated as $100 \%$. The means \pm SD from three independent experiments are shown. ${ }^{*} \mathrm{P}<0.05,{ }^{* * *} \mathrm{P}<0.01$. 

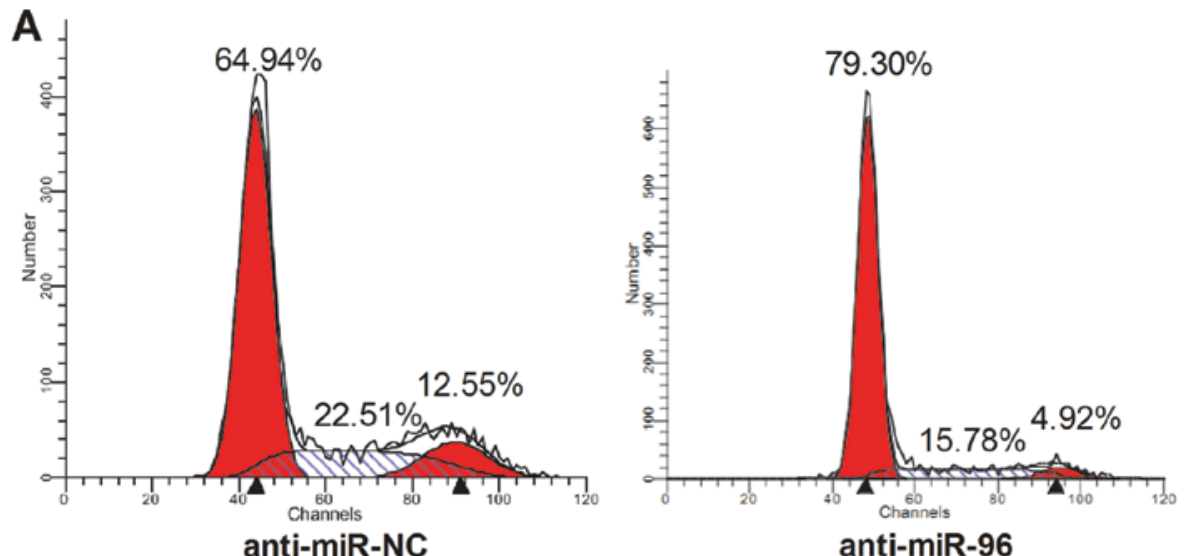

B

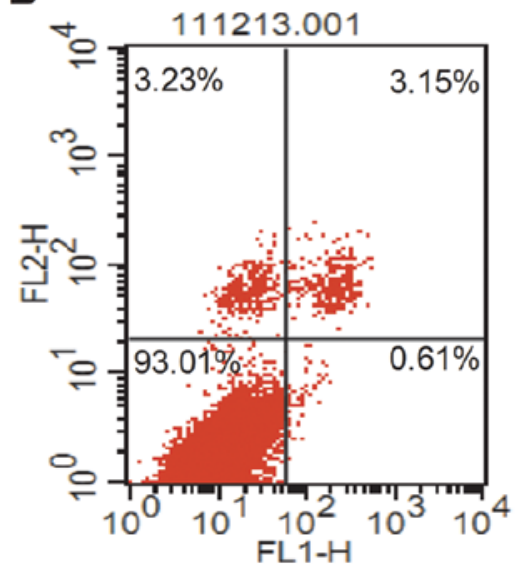

anti-miR-NC

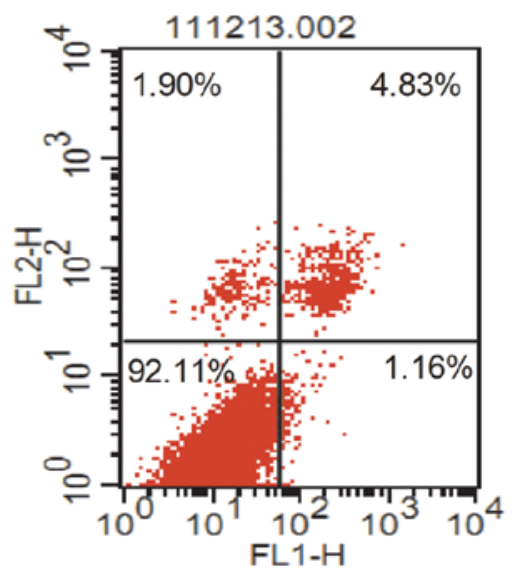

anti-miR-96

C
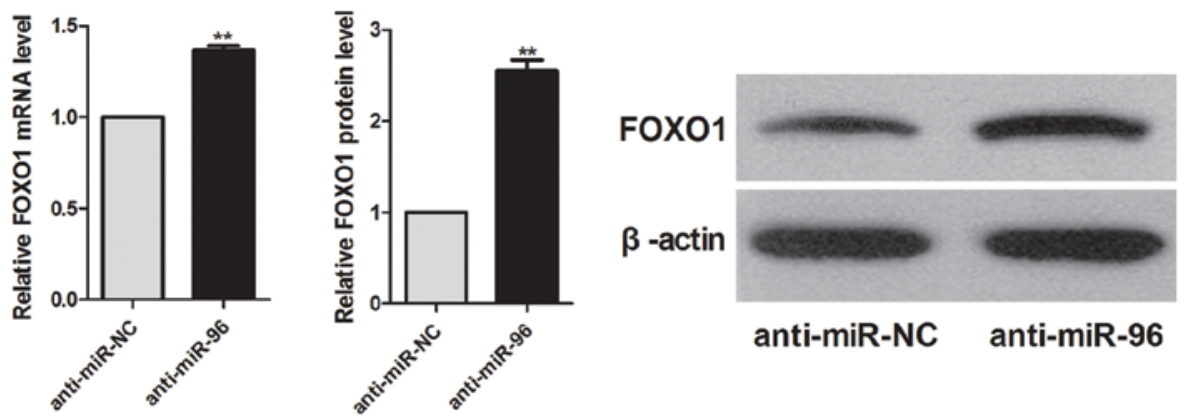

D
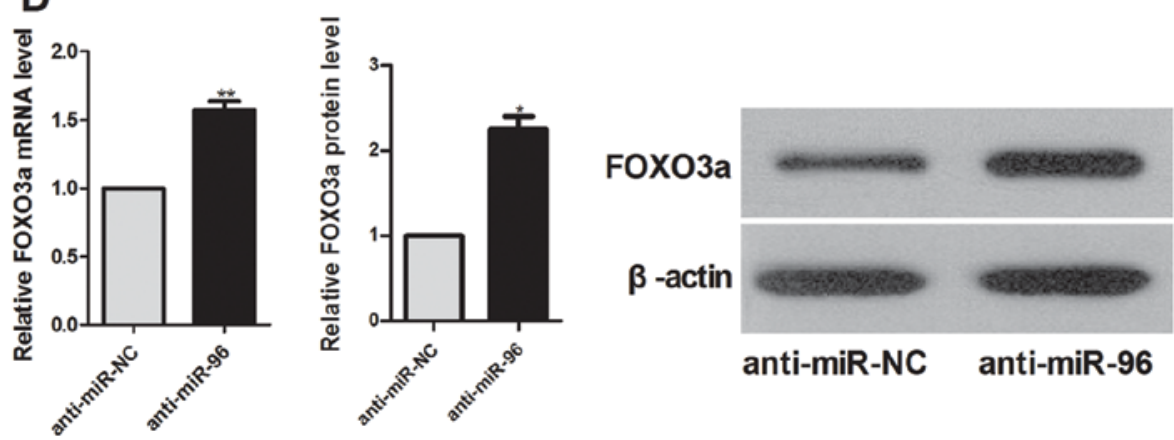

Figure 2. Effects of miR-96 on cell cycle distribution, apoptotic index and the expression of FOXO1 and FOXO3a in HepG2 cells. (A) Transfection with antimiR-96 caused cell cycle arrest. Cell-cycle analysis was conducted $72 \mathrm{~h}$ after transfection by propidium iodide staining and flow cytometry. Numbers over each histogram indicate the percentage of cells in the G0-G1, S and G2-M phases of the cell cycle. (B) Anti-miR-96 transfection did not induce cell apoptosis. Cell apoptosis in HepG2 cells was detected by Annexin V-FITC labeling combined with flow cytometry $72 \mathrm{~h}$ after transfection of cells with the anti-miRNAs. The extent of apoptosis was determined by the percentage of the total cells that were apoptotic. (C and D) the expression of FOXO1 and FOXO3a was evaluated $48 \mathrm{~h}$ after cell culture. Quantitative RT-PCR shows an increase in FOXO1 and FOXO3a mRNA levels in anti-miR-96-transfected HepG2 cells, compared with control cells. Western blot analysis shows a concomitant increase in FOXO1 and FOXO3a protein levels. The expression data were normalized to $\beta$-actin expression, and the FOXO1 and FOXO3a levels in control cells were set to 1.0 and the values in other samples were calculated relative to the levels in control cells. 
A
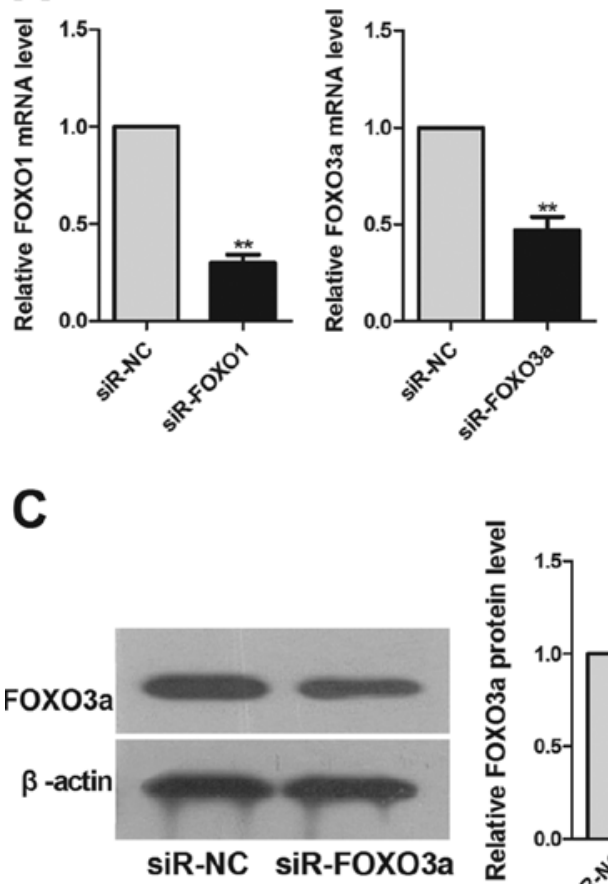

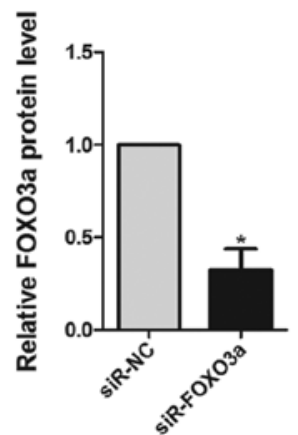

B
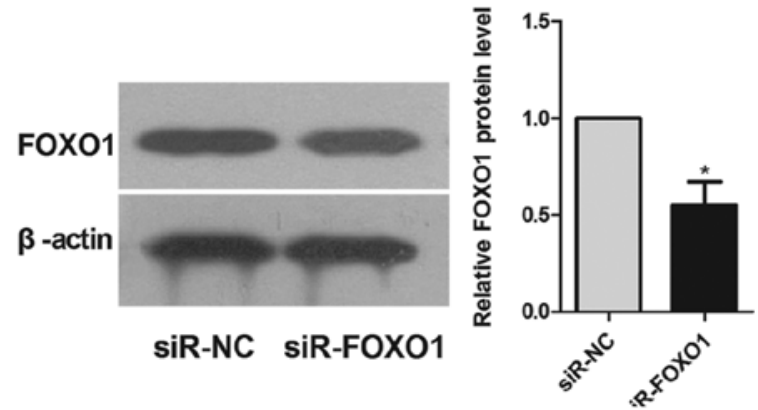

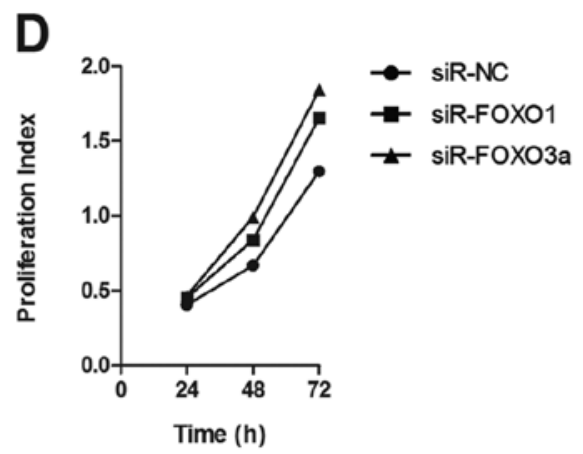

Figure 3. Inhibition of FOXO1 and FOXO3a by siRNAs in HepG2 cells and the effect of siRNAs on the proliferation of HepG2 cells. (A) Real-time PCR analysis of the FOXO1 and FOXO3a levels was performed $48 \mathrm{~h}$ after transfection with FOXO1- and FOXO3a-specific siRNAs (siR-FOXO1 and siR-FOXO3a, respectively). The data show that the expression of FOXO1 and FOXO3a was effectively inhibited by siRNAs. (B and C) Western blot analysis shows that the FOXO1 and FOXO3a protein levels were downregulated after transfection with siRNAs. The expression data were normalized to $\beta$-actin expression, the normalized FOXO1 and FOXO3a level in the negative control siRNA (siR-NC)-transfected cells was set to 1.0, and the values for other samples were calculated relative to those in the negative control. (D) The cellular proliferation rates were strongly increased in cells transfected with siR-FOXO1 and siR-FOXO3a, compared with siR-NC-transfected cells, 24,48 and $72 \mathrm{~h}$ after transfection. The means $\pm \mathrm{SD}$ from three independent experiments are shown. ${ }^{*} \mathrm{P}<0.05,{ }^{* *} \mathrm{P}<0.01$.

FOXO1 and FOXO3a modulate HepG2 cell proliferation, migration and colony formation. Transfection of HepG2 cells with siRNAs targeting FOXO1 and FOXO3a markedly promoted cell proliferation (Fig. 3D) and caused a significant increase in cell migration, compared with the corresponding negative controls (Fig. 4A). This result supports a functional role for FOXO1 and FOXO3a in mediating cell proliferation and migration in malignant hepatocytes.

We evaluated the effects of FOXO1 and FOXO3a on the clonogenicity of HepG2 cells by using a colony formation assay. Analysis of colony formation showed that downregulation of the expression of FOXO1 and FOXO3a using siRNAs resulted in the formation of much fewer and smaller colonies than in control transfectants (Fig. 4B). Inhibition of FOXO1 and FOXO3a expression also promoted the anchorage-independent growth of HepG2 cells significantly, as shown by the increase in colony number and size (Fig. 4B).

FOXO1 and FOXO3a affect cell cycle and apoptosis of HepG2 cells. The downregulation of FOXO3a or FOXO1 expression by transfection with siRNAs altered cell cycle regulation in HepG2 cells, in that the ratio of G0/G1 phase cells decreased and the fraction of S-phase cells significantly increased, compared with the cells treated with siR-NC at 72 h (Fig. 5A). Compared to the negative control, inhibition of FOXO1 and FOXO3a induced less apoptosis (Fig. 5B) in HepG2 cells.

\section{Discussion}

miRNAs are involved in critical biological processes, including development, differentiation, apoptosis, and proliferation, through imperfect pairing with target messenger RNAs of protein-coding genes and transcriptional or post-transcriptional regulation of their expression. Numerous studies have reported that miRNAs are involved in the development and progression of various types of human cancer and proposed a role for miRNAs as potential novel targets for anti-cancer therapies (18-20). The genesis and growth of HCC is intricately and multifactorially regulated, and miRNAs may be involved in this process. Several studies have reported miRNA expression profiling in human HCC cells and tissues and in experimental models of HCC (21-23). Consistently, these studies have all shown that specific miRNAs are abnormally expressed in malignant HCC cells or tissues compared to non-malignant hepatocytes or tissue. miR-96 is among the miRNAs that are abnormally increased in HCC compared to non-tumoral tissue (12).

Our present study shows that miR-96 expression was markedly upregulated in liver cancer cells, compared with that in normal liver cells, consistent with results described in a previous report (12). miR-96 may be associated with HCC and resides on chromosome $7 \mathrm{q} 32.2$ in the intergenic region between two protein-coding genes. Previously, miR-96 was identified as an HBV infection-related miRNA (24). miR-96 was also found to be upregulated in colorectal cancer (25). 
A a

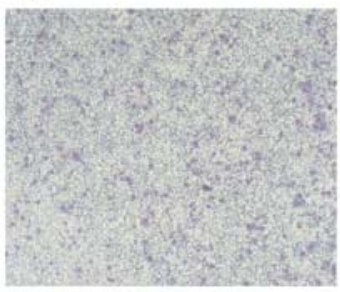

siR-NC

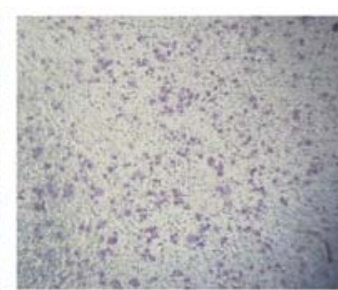

siR-FOX01

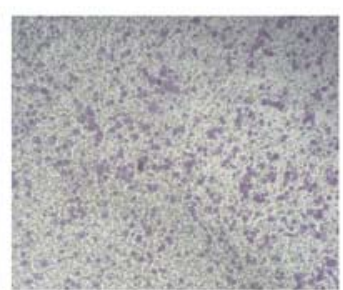

siR-FOXO3a

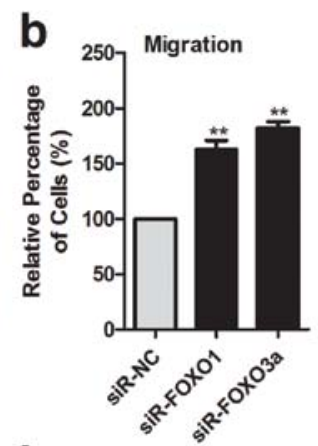

b

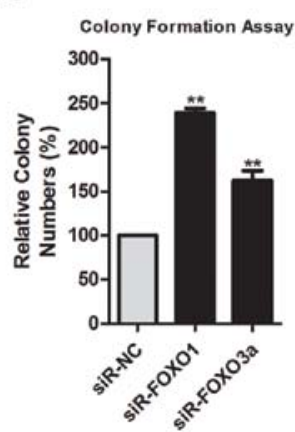

d

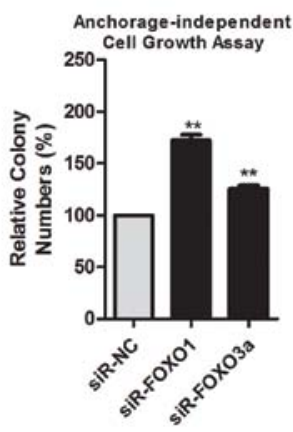

Figure 4. Effects of FOXO1 and FOXO3a inhibition on cell migration and colony formation of HepG2 cells. (Aa) The result of cell migration across an 8- $\mu \mathrm{m}$ pore size membrane without Matrigel. (Ab) Histogram indicating that the relative percentage of cells across a membrane with 8 - $\mu$ m pores without Matrigel is increased in cells transfected with FOXO1- and FOXO3a-specific siRNAs, compared with that of the siR-NC-transfected cells. The relative percentage of migrating cells from the siR-NC group was designated as $100 \%$. (Ba and c) Representative results of colony formation and anchorage-independent growth in soft agar. The numbers of colonies and their sizes are strongly increased in cells transfected with FOXO1- and FOXO3a-specific siRNAs, compared with those in the siR-NC-transfected cells. Each bar corresponds to the means \pm SD of three experiments. (Bb and d) Histograms indicate that FOXO1 and FOXO3a inhibition markedly promoted the colony formation and anchorage-independent cell growth. The percentage of colony numbers in the negative control was designated as $100 \%{ }^{* *} \mathrm{P}<0.01$.

Furthermore, a previous report demonstrated that the level of miR-96 was elevated in chronic myelocytic leukemia (CML) patients compared to the levels in healthy donor samples, indicating a higher possibility of the involvement of miR-96 in CML pathogenesis (26). In bladder urothelial carcinoma, miR-96 was the most significantly upregulated miRNA (27). Using DNA microarrays, it was demonstrated that miR-96 was significantly and consistently upregulated in non-small cell lung carcinoma cells (28).

The malignant potential of tumor cells is reflected by their increased proliferation, migration and colony formation abilities. We found that miR-96 promotes the proliferation and migration of HCC cells. Moreover, our results revealed that colonies formed in soft agar were markedly fewer and smaller when cells were treated with an inhibitor of miR-96, compared to those formed by cells treated with a negative control. A similar result was observed in the colony formation assay. These data indicate that the malignant potential of HepG2 cells is strengthened by miR-96.

miRNAs play important regulatory roles in animals and plants by targeting mRNAs for cleavage or translational repression. To explore the mechanism by which miR-96 suppressed the proliferation, migration, and clonogenicity of HCC cells, we used a bioinformatics analysis to search for genes regulated by miR-96, using TargetScan (http://www.targetscan.org/) (29) and found that miR-96 might target FOXO1 and FOXO3a, which are members of the FOXO subfamily. In our study, we demonstrated that with the inhibition of miR-96, the expression of FOXO1 and FOXO3a is upregulated. The FOXO subfamily of Forkhead transcription factors, including FOXO1 (FKHR), FOXO3a (FKHRL1), FOXO4 (AFX) and FOXO6, contains evolutionarily conserved transcriptional activators that are 
A

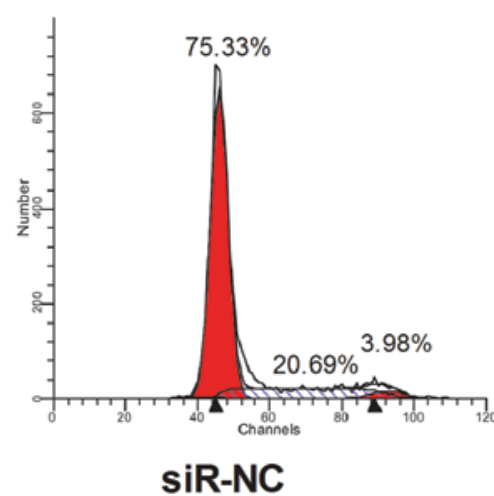

B

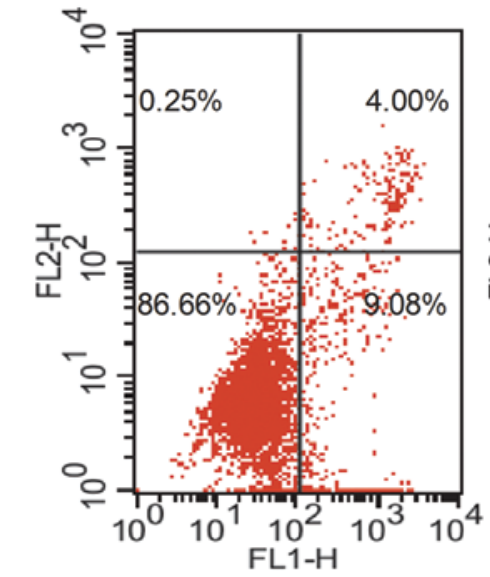

siR-NC

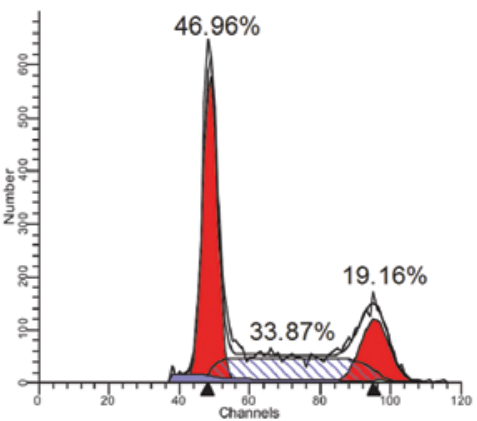

siR-FOXO1

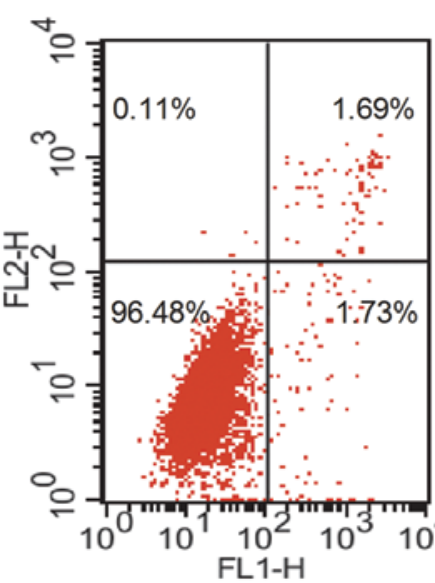

siR-FOX01

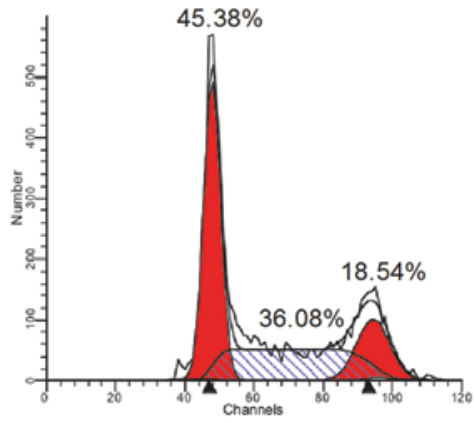

siR-FOXO3a

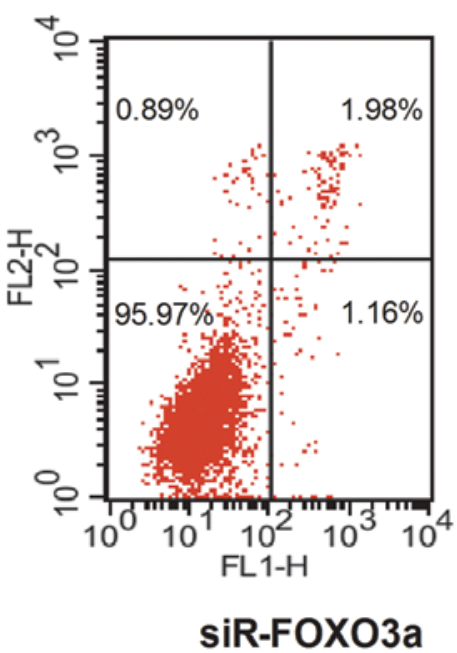

Figure 5. Effects of FOXO1 and FOXO3a inhibition on cell cycle distribution and apoptotic index in HepG2 cells. (A) Compared with siR-NC, siR-FOXO3a and siR-FOXO1 transfection affects the cell cycle. Numbers over each histogram indicate the percentage of cells in the G0-G1, S and G2-M phases of the cell cycle. (B) Compared to siR-NC, siR-FOXO1 and siR-FOXO3a transfection induces less cell apoptosis. The extent of apoptosis was determined by the percentage of the total cells that were apoptotic.

characterized by a highly conserved Forkhead domain with a DNA-binding motif (13). FOXO proteins play pivotal roles in biological processes, such as apoptosis, cell cycle control, differentiation, stress response, DNA damage repair, and glucose metabolism (30). FOXO transcription factors are considered key tumor suppressors.

The TargetScan program predicted that FOXO1 has the highest context score (an index for the strength of the bond between mature miRNA and target sites) to miR-96. FOXO1 could be the most important HCC-related target gene of miR-96. We demonstrated that inhibition of miR-96 in liver cancer cells led to the upregulation of FOXO1, indicating that miR-96 can inhibit the expression of FOXO1. However, whether miR-96 can directly target the 3'-UTR of FOXO1 mRNA remains to be confirmed in future studies.

FOXO3a is also known to control cellular differentiation, DNA repair, longevity, as well as oxidative stress. FOXO3a exerts its apoptotic effect by transactivating apoptotic factors, such as bim (a pro-apoptotic member of the Bcl-2 protein family) and Fas ligand (FasL) (31). In breast cancer cells, miR-96 was shown to likely promote breast cancer proliferation by directly targeting the 3'-UTR of the FOXO3a mRNA, consequently reducing the expression of cyclin-dependent kinase (CDK) inhibitors, and upregulating the cell cycle regulator cyclin D1 (11). In HCC cells, we demonstrated that with the reduction of
miR-96, FOXO3a was upregulated. These data suggest that in HCC cells miR-96 might directly target the 3'-UTR of FOXO3a mRNA and lead to the downregulation of FOXO3a.

Downregulation of FOXO1 in chicken embryo fibroblasts or inhibition of the transcriptional activity of FOXO3a protein in human breast cancer cells promotes cell transformation and tumor progression $(10,32)$. In human breast cancer cells, inhibition of miR-96 results in downregulation of FOXO1 and FOXO3a, and thus induces cell proliferation $(9,11)$. Our study showed that the inhibition of FOXO1 and FOXO3a caused increased cell proliferation, migration, and colony formation of HepG2 cells. The inhibition of FOXO1 and FOXO3a also caused retention of more cells in the S-phase of the cell cycle and reduction of apoptosis. Thus, miR-96 might modulate the proliferation and colony formation of HCC cells through FOXO1 and FOXO3a.

Although miR-96 can downregulate the expression of FOXO1 and FOXO3a, inhibition of miR-96 caused significant cell cycle arrest, but did not induce significant apoptosis. The mechanism underlying this process remains unknown and requires further exploration.

In conclusion, miR-96 suppresses the expression of FOXO1 and FOXO3a and promotes the proliferation, migration, and clonogenicity of liver cancer cells. Further research is needed to explore the precise molecular mechanism underlying this 
process. However, our findings suggest that upregulation of miR-96 may play an important role in promoting carcinogenesis and progression of liver cancer.

\section{Acknowledgements}

This study was supported by grants from the National Natural Science Foundation of China, no. 81101824 (X.H.) and 81000874 (C.X.).

\section{References}

1. Srivatanakul P, Sriplung H and Deerasamee S: Epidemiology of liver cancer: an overview. Asian Pac J Cancer Prev 5: 118-125, 2004

2. Lavanchy D: Hepatitis B virus epidemiology, disease burden, treatment, and current and emerging prevention and control measures. J Viral Hepat 11: 97-107, 2004

3. El-Serag HB and Mason AC: Risk factors for the rising rates of primary liver cancer in the United States. Arch Intern Med 160: 3227-3230, 2000.

4. Baan R, Straif K, Grosse Y, et al: Carcinogenicity of alcoholic beverages. Lancet Oncol 8: 292-293, 2007.

5. Girard M, Jacquemin E, Munnich A, Lyonnet S and HenrionCaude A: miR-122, a paradigm for the role of microRNAs in the liver. J Hepatol 48: 648-656, 2008.

6. O'Connell RM, Rao DS, Chaudhuri AA and Baltimore D Physiological and pathological roles for microRNAs in the immune system. Nat Rev Immunol 10: 111-122, 2010.

7. Skaftnesmo KO, Prestegarden L, Micklem DR and Lorens JB: microRNAs in tumorigenesis. Curr Pharm Biotechnol 8 : 320-325, 2007.

8. Calin GA, Sevignani C, Dumitru CD, et al: Human microRNA genes are frequently located at fragile sites and genomic regions involved in cancers. Proc Natl Acad Sci USA 101: 2999-3004, 2004.

9. Guttilla IK and White BA: Coordinate regulation of FOXO1 by miR-27a, miR-96, and miR-182 in breast cancer cells. J Biol Chem 284: 23204-23216, 2009.

10. Hu MC, Lee DF, Xia W, et al: IkappaB kinase promotes tumorigenesis through inhibition of forkhead FOXO3a. Cell 117: 225-237, 2004

11. Lin H, Dai T, Xiong H, et al: Unregulated miR-96 induces cell proliferation in human breast cancer by downregulating transcriptional factor FOXO3a. PLoS One 5: e15797, 2010.

12. Pineau P, Volinia S, McJunkin K, et al: miR-221 overexpression contributes to liver tumorigenesis. Proc Natl Acad Sci USA 107: 264-269, 2010.

13. Greer EL and Brunet A: FOXO transcription factors at the interface between longevity and tumor suppression. Oncogene 24: 7410-7425, 2005.

14. Myatt SS, Wang J, Monteiro LJ, et al: Definition of microRNAs that repress expression of the tumor suppressor gene FOXO1 in endometrial cancer. Cancer Res 70: 367-377, 2010.
15. He XX, Chang Y, Meng FY, et al: microRNA-375 targets AEG-1 in hepatocellular carcinoma and suppresses liver cancer cell grow th in vitro and in vivo. Oncogene 31: 3357-3369, 2012

16. Samarin J, Rehm M, Krueger B, Waschke J and GoppeltStruebe M: Up-regulation of connective tissue growth factor in endothelial cells by the microtubule-destabilizing agent combretastatin A-4. Mol Cancer Res 7: 180-188, 2009.

17. Zrelli H, Matsuoka M, Kitazaki S, Zarrouk M and Miyazaki $\mathrm{H}$ Hydroxytyrosol reduces intracellular reactive oxygen species levels in vascular endothelial cells by upregulating catalase expression through the AMPK-FOXO3a pathway. Eur J Pharmacol 660: 275-282, 2011.

18. Gregory RI and Shiekhattar R: microRNA biogenesis and cancer. Cancer Res 65: 3509-3512, 2005.

19. Calin GA and Croce CM: microRNA signatures in human cancers. Nat Rev Cancer 6: 857-866, 2006.

20. Esquela-Kerscher A and Slack FJ: Oncomirs - microRNAs with a role in cancer. Nat Rev Cancer 6: 259-269, 2006.

21. Murakami Y, Yasuda T, Saigo K, et al: Comprehensive analysis of microRNA expression patterns in hepatocellular carcinoma and non-tumorous tissues. Oncogene 25: 2537-2545, 2006.

22. Jiang J, Gusev Y, Aderca I, et al: Association of microRNA expression in hepatocellular carcinomas with hepatitis infection, cirrhosis, and patient survival. Clin Cancer Res 14: 419-427, 2008.

23. Huang YS, Dai Y, Yu XF, et al: Microarray analysis of microRNA expression in hepatocellular carcinoma and non-tumorous tissues without viral hepatitis. J Gastroenterol Hepatol 23: 87-94, 2008.

24. Ladeiro Y, Couchy G, Balabaud C, et al: microRNA profiling in hepatocellular tumors is associated with clinical features and oncogene/tumor suppressor gene mutations. Hepatology 47: 1955-1963, 2008.

25. Bandres E, Cubedo E, Agirre X, et al: Identification by Real-time PCR of 13 mature microRNAs differentially expressed in colorectal cancer and non-tumoral tissues. Mol Cancer 5: 29, 2006.

26. Agirre X, Jimenez-Velasco A, San Jose-Eneriz E, et al: Downregulation of hsa-miR-10a in chronic myeloid leukemia CD34 ${ }^{+}$ cells increases USF2-mediated cell growth. Mol Cancer Res 6: 1830-1840, 2008.

27. Han Y, Chen J, Zhao X, et al: microRNA expression signatures of bladder cancer revealed by deep sequencing. PLoS One 6: e18286, 2011

28. Derynck R, Akhurst RJ and Balmain A: TGF-beta signaling in tumor suppression and cancer progression. Nat Genet 29: 117-129, 2001.

29. Lewis BP, Burge CB and Bartel DP: Conserved seed pairing, often flanked by adenosines, indicates that thousands of human genes are microRNA targets. Cell 120: 15-20, 2005.

30. Huang $\mathrm{H}$ and Tindall DJ: Dynamic FoxO transcription factors J Cell Sci 120: 2479-2487, 2007.

31. Yang JY, Xia W and Hu MC: Ionizing radiation activates expression of FOXO3a, Fas ligand, and Bim, and induces cell apoptosis. Int J Oncol 29: 643-648, 2006.

32. Aoki M, Jiang $\mathrm{H}$ and Vogt PK: Proteasomal degradation of the FoxO1 transcriptional regulator in cells transformed by the $\mathrm{P} 3 \mathrm{k}$ and Akt oncoproteins. Proc Natl Acad Sci USA 101: 13613-13617, 2004. 